\section{Moments and Polyspectra of the Discrete-Time Random Telegraph Signal}

\author{
Bernard Picinbono, Fellow, IEEE
}

\begin{abstract}
The random telegraph signal (RTS) is an ordered signal which means that the explicit expression of the moment functions requires that the time instants appearing in these moments are put in an increasing order. This property greatly complicates the calculation of higher order polyspectra, Fourier transforms of moments. This correspondence presents an extension to the discrete-time (DT) case of a method already known in continuous time. This extension exhibits new problems which makes the calculation even more difficult. These difficulties are analyzed and the structure of trispectrum is discussed in detail.
\end{abstract}

Index Terms-Higher order statistical analysis, trispectrum.

\section{INTRODUCTION}

The continuous-time (CT) random telegraph signal (RTS) is one of the simplest examples of a signal constructed from a Poisson process. It plays an important role in many areas of signal processing. Its correlation function has an exponential shape and its moments of an order higher than two have a very simple mathematical expression [1]. On the other hand, the Fourier transforms (FTs) of these moments, or the polyspectra, are much more difficult to calculate, as seen in [2]. But it is often more convenient to work in the frequency domain than in the time domain. This is especially the case when using linear filtering, because products are simpler to manipulate than convolutions. This property, well known for the input-output relationship in a linear filter, is still valid when using higher order statistics and polyspectra are often more interesting to use than time moments. This justifies the interest in the knowledge of polyspectra of various signals and there is a lot of literature on the calculation of such polyspectra of continuous-time signals (see [2]) introducing sometimes various technical difficulties.

The purpose of this correspondence is to study the same problem in the discrete-time (DT) case. The calculation of the moments is quite similar, and is briefly presented in Section II. On the other hand, the calculation of the polyspectra introduces difficulties which do not appear in the CT case. As a consequence, it is almost impossible to obtain explicitly the general expression of the polyspectra. However, bispectrum and trispectrum can be calculated, and this is presented in Section III. The main purpose in this section is to stress the difference between the CT and DT cases, avoiding calculations that are simple transpositions from CT to DT. This allows us to understand the difficulties appearing in the determination of polyspectra of an order higher than the one of the trispectrum.

The result of the calculation exhibits some interesting points concerning the trispectrum. The first important result, already verified in the CT, is that the trispectrum introduces a normal spectral density on the normal manifolds of the frequency domain. This property has various practical consequences that are discussed. The second result concerns the density of the trispectrum on the stationary manifold of the frequency domain. Indeed, it is important to verify whether or not this density is bounded, as for example for the trispectrum of linear models. This point requires a specific treatment. Indeed, we shall see in the cal-

Manuscript received November 14, 1999; revised June 13, 2000.

The author is with the Laboratoire des Signaux et Systèmes, Supélec, Plateau de Moulon, 91192 Gif sur Yvette, France (e-mail: picinbono@1ss.supelec.fr).

Communicated by J. A. O'Sullivan, Associate Editor for Detection and Estimation.

Publisher Item Identifier S 0018-9448(00)09676-0. culations that it appears in the frequency-domain functions that are not bounded, which can introduce unbounded trispectral densities. However, despite this fact, a more detailed analysis shows that the trispectrum remains bounded. Finally, the correspondence gives the algebraic expression of the trispectrum which can be used in various problems of higher order statistics analysis [3]-[5].

\section{MOMENTS OF THE RTS}

The RTS is a real stationary signal $x(t), t$ integer, taking only the values \pm 1 and satisfying the recursion $x(t)=x(t-1) u(t)$, where $u(t)$ is a strictly white noise, or a sequence of independent and identically distributed (i.i.d.) random variables taking only the values +1 and -1 with probabilities $p$ and $1-p$, respectively. As a consequence, $x^{2 k}(t)=$ $1, x^{2 k+1}(t)=x(t)$, and the same relations are valid for $u(t)$. The stationarity assumption implies that $E[x(t)]=0$. It results from the recursion that

$$
x(t) x(t+\tau)=x^{2}(t) u(t+1) u(t+2) \ldots u(t+\tau)
$$

and, as a consequence, the correlation function of $x(t)$ is

$$
\gamma(\tau)=E[x(t) x(t+\tau)]=a^{|\tau|}
$$

with $a=2 p-1$. This shows that, as in the CT, the correlation function has an exponential shape. However, as $a$ can take negative values, this correlation function can have an oscillatory behavior, which is impossible in the CT case.

It results immediately from the definition that the third-order moment $E\left[x\left(t_{1}\right) x\left(t_{2}\right) x\left(t_{3}\right)\right]$ is zero.

In order to calculate the fourth-order moment, let us introduce the random variable

$$
X=x(t) x\left(t+k_{1}\right) x\left(t+k_{2}\right) x\left(t+k_{3}\right)
$$

where $0 \leq k_{1} \leq k_{2} \leq k_{3}$. We deduce from the previous properties of $x(t)$ and $u(t)$ that

$$
X=u(t+1) \ldots u\left(t+k_{1}\right) u\left(t+k_{2}+1\right) \ldots u\left(t+k_{3}\right)
$$

and this yields

$$
E(X)=a^{k_{1}} a^{k_{3}-k_{2}} .
$$

As a consequence, we have

$$
m_{4}\left(\left\{t_{i}\right\}\right)=E\left[x\left(t_{1}\right) \ldots x\left(t_{4}\right)\right]=\gamma\left(\theta_{2}-\theta_{1}\right) \gamma\left(\theta_{4}-\theta_{3}\right)
$$

where the $\theta_{i}$ 's are deduced from the $t_{i}$ 's by the ordering permutation characterized by $\theta_{i} \leq \theta_{i+1}$. Thus the explicit expression of $m_{4}\left(\left\{t_{i}\right\}\right)$ is given in terms of the ordered time instants $\theta_{i}$, which characterizes a structure of an ordered signal [2]. It is obvious that (6) can be extended to any moment of an even order and that the moments of odd orders are zero.

This shows that, as in the CT, the RTS is an ordered signal, and one can show that this property also appears for various DT Markov signals [6]. It is precisely this ordered property which greatly complicates the calculation of the polyspectra.

In many problems it is more interesting to work with cumulants than with moments [7]. At this point it is worth noting that if the expression (6) of the moments is rather simple, this is no longer the case for cumulants, because the relation between moments and cumulants makes use of a complicated transformation (see [7, p. 19]). That is why we shall reduce our calculation to moment polyspectra. 
We deduce from (6) that fourth-order moments can be expressed in terms of the correlation function. This also appears in the case of normal (or Gaussian) signals, for which there exists the famous relation

$$
\begin{aligned}
m_{4 N}\left(\left\{t_{i}\right\}\right)=\gamma\left(t_{1}-t_{2}\right) \gamma\left(t_{3}-t_{4}\right)+ & \gamma\left(t_{1}-t_{3}\right) \gamma\left(t_{2}-t_{4}\right) \\
& +\gamma\left(t_{1}-t_{4}\right) \gamma\left(t_{2}-t_{3}\right)
\end{aligned}
$$

valid whatever the order of the instants $t_{i}$. However, the difference between (6) and (7) is obvious and this suggests the question of the consequences of (6). More precisely, it is interesting to search whether or not there are other signals with moments given by (6). This is specified by the following result.

Theorem: Let $x(t)$ be a stationary and ergodic DT signal of unit power and let $\gamma(\tau)$ be its correlation function. If its fourth-order moment $m_{4}\left(\left\{t_{i}\right\}\right)$ takes the form $f\left(\theta_{2}-\theta_{1}\right) f\left(\theta_{4}-\theta_{3}\right)$, where the $\theta_{i}$ 's denote the ordered permutation of the $t_{i}$ 's, then $x(t)$ takes only the values \pm 1 and

$$
f(\tau)=\gamma(\tau)=a^{|\tau|}, \quad|a|<1 .
$$

Proof: Let $y(t)=x^{2}(t)$. As $x(t)$ is of unit power $E[y(t)]=1$. As the correlation function $\gamma_{y}(\cdot)$ of $y(t)$ is a fourth-order moment of $x(t)$, it results from the assumption that $\gamma_{y}\left(\theta_{2}-\theta_{1}\right)=f^{2}(0)$. This implies that $y(t)$ is a random variable $Y$. As $y(t)$ is ergodic, $Y$ cannot be random and is equal with probability 1 to a nonrandom constant c. But as $E(Y)=1$, this constant is equal to 1 and $Y=1$ with probability 1 . This implies that $x(t)= \pm 1$, and therefore $f^{2}(0)=1$. As $f(\cdot)$ appears only with its product, we can assume that $f(0)=1$. As a result

$$
E\left[x\left(\theta_{1}\right) x\left(\theta_{2}\right) x^{2}\left(\theta_{3}\right)\right]=E\left[x\left(\theta_{1}\right) x\left(\theta_{2}\right)\right]
$$

or

$$
f\left(\theta_{2}-\theta_{1}\right)=\gamma\left(\theta_{2}-\theta_{1}\right) .
$$

Similarly,

$$
E\left[x\left(\theta_{1}\right) x^{2}\left(\theta_{2}\right) x\left(\theta_{3}\right)\right]=E\left[x\left(\theta_{1}\right) x\left(\theta_{3}\right)\right]
$$

or

$$
\gamma\left(\theta_{2}-\theta_{1}\right) \gamma\left(\theta_{3}-\theta_{2}\right)=\gamma\left(\theta_{3}-\theta_{1}\right)
$$

This implies that $\gamma(\tau)=a^{|\tau|}$, and as $x(t)$ is stationary, $|a|<1$.

This result is interesting because it shows that the structure of the moment (6) is strongly connected with the exponential correlation function.

\section{TRISPECTRUM OF THE RTS}

As the moments of odd order are zero, the bispectrum is also zero. The trispectrum is deduced from the fourth-order spectral moment, FT of $m_{4}\left(\left\{t_{i}\right\}\right)$, defined by

$$
M(\boldsymbol{f})=\sum_{t_{1}} \sum_{t_{2}} \sum_{t_{3}} \sum_{t_{\mathbf{4}}} m_{4}(\boldsymbol{t}) \exp \left(-2 \pi j \boldsymbol{f}^{T} \boldsymbol{t}\right)
$$

where $m_{4}\left(\left\{t_{i}\right\}\right)$ is defined by (6), $\boldsymbol{f}$ is the frequency vector with components $f_{i}$, and $\boldsymbol{t}$ is the time vector with components $t_{i}$.

It is in the calculation of this expression that the main differences between the CT and the DT cases appear. This is already true when calculating simply the power spectrum of an exponential correlation function. In the CT we have

$$
\Gamma(f)=\int_{-\infty}^{+\infty} \exp (-a|\tau|) \exp (-2 \pi j f \tau) d \tau
$$

and the calculation can be reduced to an integral from 0 to $\infty$, with its complex conjugate. In the DT case we have

$$
\Gamma(f)=\sum_{-\infty}^{+\infty} a^{|\tau|} \exp (-2 \pi j f \tau), \quad \tau \text { integer }
$$

and in order to use two complex conjugate series, it is necessary to suppress the term corresponding to $\tau=0$.

The same phenomenon, though much more complicated, appears for (13), and we must distinguish between terms with distinct $t_{i}$ 's and terms where some $t_{i}$ 's are equal. This introduces five kinds of terms.

a) The term $A$ corresponds to the sum (13) where all the $t_{i}$ 's are distinct.

b) In this case there is a sum with respect to only three distinct $t_{i}$ 's. For example, if $t_{1}=t_{2}$, the term $B_{12}$ corresponds in (13) to the sum with

$$
E\left[x^{2}\left(t_{1}\right) x\left(t_{3}\right) x\left(t_{4}\right)\right]=\gamma\left(t_{3}-t_{4}\right) .
$$

There are, of course, six distinct terms $B_{i j}$ such as $B_{12}$.

c) This corresponds to two uncoupled instants such that $t_{1}=t_{2}$, $t_{3}=t_{4}$. The corresponding moment in (13) is

$$
E\left[x^{2}\left(t_{1}\right) x^{2}\left(t_{3}\right)\right]=1
$$

and there are three distinct terms $C_{i j}$.

d) The terms $D_{i}$ correspond to two coupled time instants such that $t_{1}=t_{2}=t_{3}, t_{4}$. The corresponding moment in (13) is

$$
E\left[x^{3}\left(t_{1}\right) x\left(t_{4}\right)\right]=\gamma\left(t_{1}-t_{4}\right)
$$

and there are four terms $D_{i}$.

e) Finally, there is one element of the sum (13) corresponding to $t_{1}=t_{2}=t_{3}=t_{4}$, with

$$
E\left[x^{4}\left(t_{1}\right)\right]=1 \text {. }
$$

It now remains to calculate the sum of all these terms.

\section{A. Distinct Time Instants}

The problem is to calculate the sum (13) where all the $t_{i}$ 's are distinct and where the moment $m_{4}(\boldsymbol{t})$ is given by (6). The principal difficulty in this calculation is that (6) is valid for ordered time instants, while (13) is independent of this ordering property.

To overcome this difficulty, the idea is to decompose (13) with distinct $t_{i}$ 's in a sum of 24 terms corresponding to the 24 distinct permutations of the $t_{i}$ 's. The sum associated with a given permutation can be expressed as

$$
\begin{array}{r}
S_{P}\left(\left\{\mu_{i}\right\}\right)=\sum_{\theta_{1}=-\infty}^{+\infty} \sum_{\theta_{2}=\theta_{1}+1}^{+\infty} \sum_{\theta_{3}=\theta_{2}+1}^{+\infty} \sum_{\theta_{4}=\theta_{3}+1}^{+\infty} \gamma\left(\theta_{4}-\theta_{3}\right) \gamma\left(\theta_{2}-\theta_{1}\right) \\
\times \exp \left[-2 \pi j\left(\sum_{i} \mu_{i} \theta_{i}\right)\right]
\end{array}
$$

where the frequencies $\mu_{i}$ are deduced from the frequencies $f_{i}$ by the permutation $P$ transforming the $t_{i}$ 's into the $\theta_{i}$ 's. An abbreviated calculation presented in the Appendix yields

$$
\begin{aligned}
S_{P}\left(\left\{\mu_{i}\right\}\right)=\Gamma_{+}\left(\mu_{4}\right) W\left(\mu_{3}+\mu_{4}\right) \Gamma_{+} & \left(\mu_{2}+\mu_{3}+\mu_{4}\right) \\
& \times \delta\left(\mu_{1}+\mu_{2}+\mu_{3}+\mu_{4}\right) .
\end{aligned}
$$

In this expression, $\Gamma_{+}(\mu)$ is defined by

$$
\Gamma_{+}(f)=\sum_{k=1}^{\infty} \gamma(k) \exp (-2 \pi j f k)
$$

and, as $\gamma(k)=a^{|k|}$

$$
\Gamma_{+}(f)=\frac{a}{\exp (2 \pi j f)-a} .
$$


Furthermore,

$$
W(f)=\sum_{k=1}^{\infty} \exp (-2 \pi j f k)
$$

and this quantity is the FT of the signal $w[k]=1$ for $k>0$ and zero otherwise. Let $v[k]$ be the signal Sign of $k$, equal to +1 for $k>0$ and even. It is obvious that $w[k]=(1 / 2)\{v[k]+1-\delta[k]\}$. By Fourier transformation this yields

$$
W(f)=(1 / 2)[V(f)+\delta(f)-1]
$$

where $\delta(\cdot)$ is, as in (17), the Dirac distribution, and

$$
V(f)=-j \cot (\pi f)
$$

is the FT of the signal $v[k]$. The function $V(f)$ is analogous to the principal value distribution defined as the FT of $v(t)=\operatorname{Sign}(t)$, where $t$ corresponds to the CT. Thus $V(f)$ defined by (22) is also a distribution, which means that any integral containing $V(f)$ must be considered as a Cauchy principal value integral. Note finally that $V(f)$ is an odd distribution, or that $V(f)=-V(-f)$.

By using these definitions, (17) can be expressed in a form that is more convenient for what follows, or

$$
\begin{aligned}
S_{P}\left(\left\{\mu_{i}\right\}\right)= & (1 / 2) \Gamma_{+}\left(\mu_{2}\right) \Gamma_{+}\left(\mu_{4}\right) \delta\left(\mu_{1}+\mu_{2}\right) \delta\left(\mu_{3}+\mu_{4}\right) \\
& -(1 / 2) V\left(\mu_{1}+\mu_{2}\right) \Gamma_{+}\left(-\mu_{1}\right) \Gamma_{+}\left(-\mu_{1}-\mu_{2}-\mu_{3}\right) \\
& \times \delta\left(\mu_{1}+\mu_{2}+\mu_{3}+\mu_{4}\right)-(1 / 2) \Gamma_{+}\left(-\mu_{1}\right) \\
& \times \Gamma_{+}\left(-\mu_{1}-\mu_{2}-\mu_{3}\right) \delta\left(\mu_{1}+\mu_{2}+\mu_{3}+\mu_{4}\right) .
\end{aligned}
$$

The first two terms of the right-hand side of this equation are the same as those obtained in the CT (see [2, eq. (48)]).

Finally, the contribution of the sum over distinct $t_{i}$ 's in (13) is obtained by making the sum of 24 terms such that (23) corresponds to the 24 permutations of the frequencies $f_{i}$. This will be done later.

\section{B. Terms $B_{i j}$}

The term $B_{12}$ is defined by

$$
\begin{aligned}
B_{12}(\boldsymbol{f})=\sum_{t_{1}} \sum_{t_{3}} & \sum_{t_{\mathbf{4}}} \gamma\left(t_{4}-t_{3}\right) \\
& \times \exp \left\{-2 \pi j\left[\left(f_{1}+f_{2}\right) t_{1}+f_{3} t_{3}+f_{4} t_{4}\right]\right\}
\end{aligned}
$$

in which the $t_{i}$ 's are distinct. The short calculations presented in the Appendix show that

$$
\begin{aligned}
B_{12}(\boldsymbol{f})=\left[\Gamma\left(f_{4}\right)-1\right] \delta\left(f_{1}\right. & \left.+f_{2}\right) \delta\left(f_{3}+f_{4}\right) \\
& -\left[\Gamma\left(f_{3}\right)+\Gamma\left(f_{4}\right)-2\right] \delta\left(\sum f_{i}\right) .
\end{aligned}
$$

In this expression, $\Gamma(f)$ is the power spectrum of the signal defined by

$$
\Gamma(f)=\sum \gamma(k) \exp (-2 \pi j k f) .
$$

There are six distinct such terms: $B_{12}, B_{13}, B_{14}, B_{23}, B_{24}, B_{34}$.

\section{Terms $C_{i j}$}

The term $C_{12}$ is defined by

$$
C_{12}(\boldsymbol{f})=\sum_{t_{1}} \sum_{t_{3} \neq t_{1}} \exp \left\{-2 \pi j\left[\left(f_{1}+f_{2}\right) t_{1}+\left(f_{3}+f_{4}\right) t_{3}\right]\right\}
$$

and its value is, of course,

$$
C_{12}(\boldsymbol{f})=\delta\left(f_{1}+f_{2}\right) \delta\left(f_{3}+f_{4}\right)-\delta\left(\sum f_{i}\right) .
$$

There are three different such terms: $C_{12}, C_{13}, C_{14}$.

\section{Terms $D_{i}$}

The term $D_{4}$ is defined by

$$
\begin{aligned}
D_{4}(\boldsymbol{f})=\sum_{t_{1}} \sum_{t_{4} \neq t_{1}} \gamma\left(t_{1}-t_{4}\right) \\
\quad \times \exp \left\{-2 \pi j\left[\left(f_{1}+f_{2}+f_{3}\right) t_{1}+f_{4} t_{4}\right]\right\}
\end{aligned}
$$

and its value is

$$
D_{4}(\boldsymbol{f})=\left[\Gamma\left(f_{4}\right)-1\right] \delta\left(\sum f_{i}\right) .
$$

There are four distinct such terms: $D_{1}, D_{2}, D_{3}, D_{4}$.

\section{E. Term E}

This term corresponding to equal $t_{i}$ 's is, of course,

$$
E(\boldsymbol{f})=\delta\left(\sum f_{i}\right)
$$

and there is only one such term.

The trispectrum is obtained by the sum of all the previous terms. In order to better understand its structure, it is necessary to make this sum by conveniently grouping the terms with similar forms.

\section{Structure of the TRISPECTRUM}

All the previous equations show that the fourth-order spectral moment $M(\boldsymbol{f})$ given by (13) is zero outside the stationary manifold defined by $f_{1}+f_{2}+f_{3}+f_{4}=0$. This is obviously a consequence of the stationarity of the RTS $x(t)$, and can be expressed as

$$
M_{4}(\boldsymbol{f})=\Gamma_{3}\left(f_{1}, f_{2}, f_{3}\right) \delta\left(f_{1}+f_{2}+f_{3}+f_{4}\right)
$$

which defines the trispectrum $\Gamma_{3}\left(f_{1}, f_{2}, f_{3}\right)$ as the density of the spectral moment on the stationary manifold. However, this density is not necessarily bounded because some terms of $M(\boldsymbol{f})$ contain two Dirac terms $\delta(\cdot)$, which introduces distributions on submanifolds of the stationary manifold. In our case, these submanifolds are only the normal manifolds (see [1] and [2]) and we shall first calculate the corresponding density.

\section{A. Distribution on the Normal Manifolds}

The first normal manifold (FNM) is defined by

$$
f_{1}+f_{2}=0 \quad f_{3}+f_{4}=0
$$

and there are two other distinct similar manifolds defined by replacing the first equation of (33) either by $f_{1}+f_{3}=0$ or by $f_{1}+f_{4}=0$.

Let us first calculate the contribution to the FNM of the term of (23) containing a product of two Dirac distributions. There are eight terms containing the product $\delta\left(f_{1}+f_{2}\right) \delta\left(f_{3}+f_{4}\right)$, and the sum of the corresponding coefficients $(1 / 2) \Gamma_{+}\left(\mu_{2}\right) \Gamma_{+}\left(\mu_{4}\right)$ can easily be written as

$$
S_{A}=\left[\Gamma_{+}\left(f_{1}\right)+\Gamma_{+}\left(f_{2}\right)\right]\left[\Gamma_{+}\left(f_{3}\right)+\Gamma_{+}\left(f_{4}\right)\right] .
$$

But it results from (18) and (26) that

$$
\begin{aligned}
\Gamma(f) & =\Gamma_{+}(f)+\Gamma_{+}^{*}(f)+1 \\
\Gamma_{+}(-f) & =\Gamma_{+}^{*}(f) .
\end{aligned}
$$

Noting that on the FNM $f_{2}=-f_{1}$ and $f_{3}=-f_{4}$, and using the fact that $\Gamma(f)$ is an even function, we deduce that

$$
S_{A}=\Gamma\left(f_{1}\right) \Gamma\left(f_{3}\right)-\Gamma\left(f_{1}\right)-\Gamma\left(f_{3}\right)+1 .
$$

As a result of (25), the terms $B_{i j}$ introducing a contribution on the FNM are $B_{12}$ and $B_{34}$, and this contribution is

$$
S_{B}=\Gamma\left(f_{1}\right)+\Gamma\left(f_{3}\right)-2 .
$$


The term $C_{12}$ introduces a contribution equal to 1 . By adding all these results we deduce that the contribution to $M(f)$ of the FNM is simply

$$
T_{1 N M}=\Gamma\left(f_{1}\right) \Gamma\left(f_{3}\right) \delta\left(f_{1}+f_{2}\right) \delta\left(f_{3}+f_{4}\right)
$$

where $\Gamma(f)$ is deduced from (19) and (35) which give

$$
\Gamma(f)=\frac{1-a^{2}}{1+a^{2}-2 a \cos (2 \pi f)} .
$$

This shows that the density on the FNM is simply the product of power spectra $\Gamma\left(f_{1}\right) \Gamma\left(f_{3}\right)$, and this is called a normal density. The same result is, of course, valid for the other two normal manifolds.

This is a very important point and it is related to various properties of the RTS such as ergodicity [8] and asymptotic normality (the Central Limit Theorem) [2]. Furthermore, it has a direct consequence on the calculation of fourth-order spectral cumulants. Indeed, this cumulant is obtained by subtracting from $M(\boldsymbol{f})$ the contribution of normal manifolds (NMs) with normal density given by (39). As a consequence, there is no contribution of the NMs to the spectral cumulant, which is one of the most important advantages of cumulants with respect to moments.

It is worth pointing out that the same result is valid for the continuous-time RTS, but the calculation is much simpler, because the terms $B, C$, and $D$ do not appear in the CT case.

\section{B. Bounded Density on the Stationary Manifold}

All the other terms of $M(\boldsymbol{f})$ defined by (13) contain only one distribution delta, or $\delta\left(\sum f_{i}\right)$, characterizing the stationarity of the signal. The only term introducing an unbounded density on the stationary manifold is the one containing $V\left(\mu_{1}+\mu_{2}\right)$ in (23). Thus we now consider the sum of all the terms previously calculated and containing only the distribution $\delta\left(\sum f_{i}\right)$, except the terms where $V(\cdot)$ appears.

In (23), we have to calculate the sum with respect to all the 24 permutations of the frequencies $f_{i}$ of the terms

$$
(-1 / 2) \Gamma_{+}\left(-\mu_{1}\right) \Gamma_{+}\left(-\mu_{1}-\mu_{2}-\mu_{3}\right)=(-1 / 2) \Gamma_{+}^{*}\left(\mu_{1}\right) \Gamma_{+}\left(\mu_{4}\right) .
$$

This sum is, of course, unchanged if we replace $\Gamma_{+}^{*}\left(\mu_{1}\right) \Gamma_{+}\left(\mu_{4}\right)$ by $\Gamma_{+}\left(\mu_{1}\right) \Gamma_{+}^{*}\left(\mu_{2}\right)$. The sum with respect to the following four permutations

$$
\begin{array}{llll}
f_{1} & f_{2} & f_{3} & f_{4} \\
f_{1} & f_{2} & f_{4} & f_{3} \\
f_{2} & f_{1} & f_{3} & f_{4} \\
f_{2} & f_{1} & f_{4} & f_{3}
\end{array}
$$

gives the contribution $A_{12}$ with

$$
A_{i j}\left(f_{i}, f_{j}\right)=-2 \operatorname{Re}\left[\Gamma_{+}\left(f_{i}\right) \Gamma_{+}^{*}\left(f_{j}\right)\right]
$$

where Re means the real part. The sum with respect to the 24 permutations of the $f_{i}$ 's yields a sum of six terms like $A_{i j}, i<j$.

The contribution of $B_{12}(\boldsymbol{f})$ defined by (25) to the stationary manifold is $-\left[\Gamma\left(f_{3}\right)+\Gamma\left(f_{4}\right)-2\right]$. The sum of the six terms $B_{i j}, i<j$ yields, therefore, the contribution $12-\sum_{i=1}^{4} \Gamma\left(f_{i}\right)$. Following the same procedure for the terms $C, D$, and $F$ we find that the bounded density on the stationary manifold can be expressed as

$$
T_{S}(\boldsymbol{f})=6-2 \sum_{i=1}^{4} \Gamma\left(f_{i}\right)-2 \sum_{i=1}^{3} \sum_{j>i}^{4} A_{i j}\left(f_{i}, f_{j}\right)
$$

where $A_{i j}\left(f_{i}, f_{j}\right)$ is defined by (42).
This sum is completely different from the one obtained in the CT case, and this is quite natural. This fact already appears for the power spectrum which is given by (40) in the DT case while the FT of the exponential correlation function $\exp (-a|\tau|)$ in the CT case is $2 a\left(a^{2}+\right.$ $\left.4 \pi f^{2}\right)^{-1}$.

\section{Contribution of the Distribution $V(\cdot)$}

This distribution appears in (23) and requires a specific analysis. Indeed, as a result of (22), $V(f)$ becomes infinite for $f \rightarrow 0$, and the sum of all the terms containing $V(f)$ in (23) can introduce an unbounded density on the stationary manifold. In order to verify whether or not this is the case, it is necessary to calculate the sum of the 24 different terms containing $V(f)$ in (23) and associated with the 24 permutations of the frequencies $f_{i}$.

For this purpose, let us consider the sum of terms containing the function $V\left(f_{1}+f_{2}\right)$. These terms are coming from the eight permutations

$$
\begin{array}{llllllll}
f_{1} & f_{2} & f_{3} & f_{4} & f_{3} & f_{4} & f_{1} & f_{2} \\
f_{2} & f_{1} & f_{3} & f_{4} & f_{3} & f_{4} & f_{2} & f_{1} \\
f_{1} & f_{2} & f_{4} & f_{3} & f_{4} & f_{3} & f_{1} & f_{2} \\
f_{2} & f_{1} & f_{4} & f_{3} & f_{4} & f_{3} & f_{2} & f_{1}
\end{array}
$$

because on the stationary manifold $f_{1}+f_{2}=-\left(f_{3}+f_{4}\right)$. Each permutation of (44) introduces the term

$$
(-1 / 2) V\left(\mu_{1}+\mu_{2}\right) \Gamma_{+}^{*}\left(\mu_{1}\right) \Gamma_{+}\left(\mu_{4}\right) .
$$

Taking $\Gamma_{+}\left(f_{i}\right)=R_{i}$ we see that the sum with respect to the four permutations of the lhs of (44) introduces the term

$$
(-1 / 2) V\left(f_{1}+f_{2}\right)\left(R_{1}+R_{2}\right)^{*}\left(R_{3}+R_{4}\right)
$$

while the remaining permutations yield

$$
(-1 / 2) V\left(f_{3}+f_{4}\right)\left(R_{1}+R_{2}\right)\left(R_{3}+R_{4}\right)^{*} \text {. }
$$

Using the fact that $V(f)$ is odd, the contribution of the eight permutations of (44) can be written $j V\left(f_{1}+f_{2}\right) \operatorname{Im}(T)$, where $\operatorname{Im}(\cdot)$ means the imaginary part and

$$
T=\left(R_{1}+R_{2}\right)\left(R_{3}+R_{4}\right)^{*} \text {. }
$$

By using the definition (22), we find that the term coming from $V\left(f_{1}+f_{2}\right)$ in the trispectrum can be expressed as

$$
S(\boldsymbol{f})=\cot \left(f_{1}+f_{2}\right) \operatorname{Im}(T)
$$

and the problem is to study its behavior when $f_{1}+f_{2} \rightarrow 0$ where the $\cot (\cdot)$ term becomes infinite. For this purpose, let us write $f_{2}=$ $-f_{1}+\epsilon$, which on the stationary manifold implies $f_{4}=-f_{3}-\epsilon$. The term $S(\boldsymbol{f})$ of (46) is now $S\left(f_{1}, f_{3}, \epsilon\right)$ and the problem is to study its behavior for $\epsilon \rightarrow 0$. From a calculation presented in the Appendix we deduce that

$$
\lim _{\epsilon \rightarrow 0} S\left(f_{1}, f_{2}, \epsilon\right)=C\left(f_{1}\right)+C\left(f_{3}\right)
$$

with

$$
C(f)=[\Gamma(f)-1] b(f)
$$

and $b(f)$ is the imaginary part of the derivative of $\Gamma_{+}(f)$ given by

$$
b(f)=2 \pi a \frac{2 a-\left(1+a^{2}\right) \cos (2 \pi f)}{\left[1+a^{2}-2 a \cos (2 \pi f)\right]^{2}} .
$$


This shows that, in spite of the presence of terms like $V\left(f_{1}+f_{2}\right)$, the trispectrum remains bounded on the stationary manifold of the frequency domain. This situation also appears in the CT domain, but with entirely different values of the trispectrum.

\section{CONCLUSION}

It results from the previous discussion that the fourth-order spectral moment (13) of the DT random telegraph signal is distributed on the normal manifold with a normal density, product of power spectra. The remaining part of the spectral moment is bounded on the stationary manifold and its value is given by (43), (46), and (47).

It is clear that the main difficulty of this calculation is due to the necessity to distinguish in (13) the terms with distinct $t_{i}$ 's and terms where some $t_{i}$ 's are equal. The calculation remains possible for the trispectrum, but it appears extremely tedious for polyspectra of orders higher than 3 , and this difficulty does not appear for the continuous time, where polyspectra of any order of the random telegraph signal have been calculated [2].

\section{APPENDIX}

\section{A. Calculation of (17)}

The sum with respect to $\theta_{4}$ in (16) yields

$$
S_{4}=e^{\left(-2 \pi j \mu_{4} \theta_{3}\right)} \Gamma_{+}\left(\mu_{4}\right) .
$$

The sum with respect to $\theta_{3}$ becomes

$$
S_{3}=\sum_{\theta_{2}+1}^{+\infty} e^{\left[-2 \pi j\left(\mu_{3}+\mu_{4}\right) \theta_{3}\right]}=e^{\left[-2 \pi j\left(\mu_{3}+\mu_{4}\right) \theta_{2}\right]} W\left(\mu_{3}+\mu_{4}\right)
$$

where $W(f)$ is defined by (20) or (21). The sum with respect to $\theta_{2}$ yields

$$
S_{2}=e^{\left[-2 \pi j\left(\mu_{2}+\mu_{3}+\mu_{4}\right){ }^{\theta}\right]} \Gamma_{+}\left(\mu_{2}+\mu_{3}+\mu_{4}\right)
$$

where $\Gamma_{+}(\cdot)$ is defined by (18) or (19), and the sum with respect to $\theta_{1}$ yields $\delta\left(\sum \mu_{i}\right)=\delta\left(\sum f_{i}\right)$

\section{B. Calculation of (25)}

The sum with respect to $t_{4}$ can be written

$$
B_{1}=\sum_{t_{\mathbf{4}}, t_{\mathbf{4}} \neq t_{1}, t_{\mathbf{4}} \neq t_{3}} \gamma\left(t_{4}-t_{3}\right) e^{\left[-2 \pi j f_{\mathbf{4}}\left(t_{4}-t_{3}\right)\right]} e^{\left(-2 \pi j f_{4} t_{3}\right)} .
$$

This gives

$$
B_{1}=e^{\left(-2 \pi j f_{4} t_{3}\right)}\left[\Gamma\left(f_{4}\right)-\gamma\left(t_{1}-t_{3}\right) e^{\left[-2 \pi j f_{4}\left(t_{1}-t_{3}\right)\right]}-1\right] .
$$

The sum with respect to $t_{3}$ becomes

$$
B_{2}=\sum_{t_{3}, t_{3} \neq t_{1}} B_{1} e^{\left(-2 \pi j f_{3} t_{3}\right)} .
$$

Inserting the previous expression of $B_{1}$ yields

$$
\begin{aligned}
B_{2}=\left[\Gamma\left(f_{4}\right)-1\right]\left[\delta\left(f_{3}+f_{4}\right)\right. & \left.-e^{\left[-2 \pi j\left(f_{3}+f_{4}\right) t_{1}\right]}\right] \\
& -\left[\Gamma\left(f_{3}\right)-1\right] e^{\left[-2 \pi j\left(f_{3}+f_{4}\right) t_{1}\right]} .
\end{aligned}
$$

It remains to make the sum with respect to $t_{1}$, that gives (25).

\section{Calculation of (47)}

It results from the definition of $R$ that $T$ defined by (45) is

$$
T=\left[\Gamma_{+}\left(f_{1}\right)+\Gamma_{+}\left(f_{2}\right)\right]\left[\Gamma_{+}\left(-f_{3}\right)+\Gamma_{+}\left(-f_{4}\right)\right] .
$$

By using the definitions given before (47), this yields

$$
T=\left[\Gamma_{+}\left(f_{1}\right)+\Gamma_{+}\left(-f_{1}+\epsilon\right)\right]\left[\Gamma_{+}\left(-f_{3}\right)+\Gamma_{+}\left(f_{3}+\epsilon\right)\right] .
$$

Let use write the derivative of $\Gamma_{+}(f)$ in the form

$$
\Gamma_{+}^{\prime}(f)=a(f)+j b(f)
$$

The first bracket of (58) becomes

$$
[]=\Gamma_{+}\left(f_{1}\right)+\Gamma_{+}\left(-f_{1}\right)+\epsilon\left[a\left(-f_{1}\right)+j b\left(-f_{1}\right)\right]+o(\epsilon) .
$$

By using (35) we get

$$
\left.[]=\left[\Gamma\left(f_{1}\right)-1+\epsilon a\left(-f_{1}\right)\right]+j \epsilon b\left(-f_{1}\right)\right]+o(\epsilon) .
$$

The same procedure can be applied to the second bracket of (58) and this yields

$$
\operatorname{Im}(T)=\epsilon\left\{b\left(f_{3}\right)\left[\Gamma\left(f_{1}\right)-1\right]+b\left(-f_{1}\right)\left[\Gamma\left(f_{3}\right)-1\right]\right\}+o(\epsilon)
$$

which gives (47). It remains to calculate $b(f)$. This is obvious from (59) and the definition (19) of $\Gamma_{+}(f)$

\section{REFERENCES}

[1] B. Picinbono, Random Signals and Systems. Englewood Cliffs, NJ: Prentice-Hall, 1993.

[2] — "Polyspectra of ordered signals," IEEE Trans. Inform. Theory, vol. 45, pp. 2239-2252, Nov. 1999.

[3] D. Brillinger, "An introduction to polyspectra," Ann. Math. Statist., vol. 36, pp. 1351-1374, 1965

[4] M. V. Hinich and H. Messer, "On the principal domain of the discrete bispectrum," IEEE Trans. Signal Processing, vol. 43, pp. 2130-2134, 1995.

[5] J. W. Dalle Molle and M. V. Hinich, "Trispectral analysis of stationary random times series," J. Acoust. Soc. Amer., vol. 97, pp. 2963-2978, 1995.

[6] B. Picinbono, "Polyspectres des signaux markoviens," in Colloque GRETSI, Vannes, France, Sept. 1999, pp. 491-494.

[7] D. Brillinger, Time Series Analysis. New York: Holt, Rinehart and Winston, 1975.

[8] B. Picinbono, "Ergodicity and fourth-order spectral moments," IEEE Trans. Inform. Theory, vol. 43, pp. 1273-1276, July 1997. 